\title{
CURRENT TOPICS IN ASSISTED REPRODUCTION IN THE CZECH REPUBLiC
}

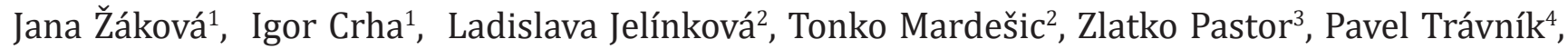 \\ Bartosz Kempisty ${ }^{1,5,6}$, Pavel Ventruba ${ }^{1}$, Michal Ješeta ${ }^{1 *}$
}

\begin{abstract}
Assisted reproduction is a very dynamic part of reproductive gynaecology with fast changes in therapeutic approaches resulting from intensive research supported by private or public companies. This progress brings also new questions regarding ethical, legal or therapeutic issues. At the $26^{\text {th }}$ Czech-Slovak symposium of assisted reproduction in Brno, several interesting topics of assisted reproduction were presented. During two days, more than 350 specialists discussed current topics related to gynaecology, embryology, andrology, genetics and sexology. From this fruitful discussion we chose the following topics that we consider to be the most important: (1) "Soft" versus "standard" stimulation protocols for IVF (2) Use of exogenous and endogenous progestins in IVF stimulation protocols (3) Cryopreservation and transplantation of ovarian tissue - state of the art and situation in the Czech Republic (4) Selection of spermatozoa with fragmented DNA: practical recommendations (5) Artificial activation of oocytes (8) Access of single and lesbian women to assisted reproduction technologies and possibility of their reproduction in the Czech Republic. Panel conclusions were presented at the end of the conference, which had great attendance, invoked lively commentaries and produced some definitive consensus. Certain issues remained inconclusive and these matters will be the subject of further discussion in future. This is a summary of the most important theses from this field in the Czech Republic in 2017.
\end{abstract}

Running title: Assisted reproduction in Czech Republic

Keywords: assisted reproduction, spermatozoa, ovarian stimulation, progestins, IVF, oocyte, embryo, oocyte activation, soft stimulation

\footnotetext{
${ }^{1}$ Department of Obstetrics and Gynecology, University Hospital and Masaryk University, Brno, Czech Republic

${ }^{2}$ Sanatorium Pronatal, Prague, Czech Republic

${ }^{3}$ Department of Obstetrics and Gynecology, 2nd Medical Faculty, Charles University Prague

${ }^{4}$ Repromeda, Brno, Czech Republic

${ }^{5}$ Department of Histology and Embryology, University of Medical Sciences, Poznan, Poland

${ }^{6}$ Department of Anatomy, University of Medical Sciences, Poznan, Poland

* Correspondence: Jeseta.Michal@fnbrno.cz

Full list of author information is available at the end of article
} 


\section{Introduction}

The first "test-tube baby" in the Czech Republic and at the same time in Central and Eastern Europe was born in the Department of Obstetrics and Gynaecology in Brno in 1982. Only four years after generally known Luise Brown. Since then, the methods, techniques and possibilities of assisted reproduction have made a major progress and have become an integral part of the reproductive medicine. There are 42 centers for infertility treatment in the Czech Republic at this time. Since 1991, an annual Symposium of assisted reproduction is held in Brno where all the Czech assisted reproduction „was born". At this platform, clinical and laboratory experiences of gynaecologists, embryologists, andrologists, geneticists, urologists, lawyers, psychologists etc. are lectured and discussed every year. The aim of this contribution is to provide a short overview of the most important topics of the $26^{\text {th }}$ Czech-Slovak symposium of assisted reproduction in 2017.

\section{"Soft" versus "standard" stimulation protocols for IVF}

The methods of ovarian stimulation have been evolving since the early time of IVF in the search for the best protocol. In general, a good stimulation protocol is one that produces a homogenous cohort of mature oocytes, with the least inconvenience and risk to the patient, not disturbing the luteal phase and resulting in the birth of a healthy singleton.

Aggressive ovarian stimulation (especially long gonadotropin-releasing hormone agonist pituitary suppression regimen) may influence the patient's wellbeing by increasing the risk of ovarian hyperstimulation syndrome, as well as it may affect the endometrium and the ovaries. Continuously increasing efficiency of IVF laboratory procedures, leading to decreased numbers of embryos transferred (preferably elective single-embryo transfer), has reduced the need for large quantities of oocytes. As a consequence, mild ovarian stimulation protocols were introduced as cost-effective, patient-friendly regimens possibly optimizing the balance between outcomes and risks of the treatment [1]. Meanwhile, many possibilities were introduced into clinical practice on how to eliminate the risk of severe hyperstimulation syndrome and how to deal with the effects of high steroid levels on implantation [2]. Nowadays, there is a clinical evidence of a strong association between the number of collected eggs and the live birth rate with increasing number of eggs up to the number of 15 , while cumulative pregnancy rates per oocyte pick-up are steadily increasing with the number of retrieved oocytes [3]. This evidence, suggesting that the number of collected eggs is a robust surrogate outcome for clinical success, clearly favours standard stimulation protocols in daily practice. However, there is no doubt that there are subgroups of patients who benefit from mild stimulation protocols (possibly advanced maternal age, women with poor ovarian reserve), but more studies are needed [4] to further assess the exact role of various mild stimulation approaches.

\section{Useofexogenousandendogenous progestins in IVF stimulation protocols}

Progestins are effective in blocking the LH surge during ovarian stimulation and can be used instead of standard gonadotropin releasing hormone analogues. Their adverse effect on the endometrial receptivity obstructs the embryo implantation in the same cycle. Such protocol can be used for stimulation of eggs donors and in cycles with planned total freezing of all eggs or embryos - in cancer patients, in case of preimplantation genetic examination of the trophoblast and social freezing. According to current experiences, progestins do not affect the number and quality of collected eggs and obtained embryos. Progestins can be used in a variety of stimulation protocols - progestin primed follicular phase stimulation, luteal phase stimulation with endogenous progesterone, double stimulation in follicular and luteal phase of the same cycle - „duostim“in low responders.

Their use opens new possibilities in ovarian stimulation protocols and improves their flexibility.

\section{Cryopreservation and transplantation of ovarian tissue - state of the art and situation in the Czech Republic}

Improved success of cancer treatment has increased the number of cancer survivors. Fertility preservation in young women before or during cancer treatment has become an important health issue. One of the strategies towards fertility preservation is cryopreservation and transplantation of ovarian tissue. This method resulted in 86 deliveries and 9 ongoing pregnancies worldwide, with increase of these numbers every month. Compared to classical methods - oocyte and/or embryo cryopreservation - ovarian tissue transplantation allows not only fertility preservation, but also restoration of endocrine function or induction of puberty. Moreover, after orthotopic transplantation, a high number of women can conceive naturally. In contrast to oocyte punction, ovarian tissue biopsy could be performed immediately, without any hormonal pre-treatment, which is the only alternative for children. Efficiency of ovarian tissue autotransplantation has reached promising levels, the cumulative pregnancy and live birth rate was $57.5 \%$ and the endocrine restoration rate was $63.9 \%$ [5].

In the Czech Republic, only eight of 42 IVF centers offer the ovarian biopsy and cryopreservation. The first transplantation of ovarian tissue was 
performed in 2015 at Sanatorium Pronatal, Prague [6] resulting in restoration of endocrine function and 17 months after the transplantation, the patient conceived naturally. The ovarian tissue was cryopreserved using classical slow freezing method and commercially available media for embryo cryopreservation. After biopsy, the ovarian tissue was transported to laboratory at approx. $4^{\circ} \mathrm{C}$ in HEPES buffered HTF. Before freezing, medulla was removed and cortical strips were prepared, $5 \times 20 \mathrm{~mm}$ each. All manipulation steps were performed at approx. $4^{\circ} \mathrm{C}$. Thawing was performed at room temperature, according to manufacturer's instructions. Three cortical strips were transplanted orthotopically [6].

Ovarian tissue transplantation is a promising method for fertility preservation. The risk of re-induction of malignant disease could be eliminated by transplantation of isolated primordial follicles only.

Constant progress in research will bring higher effectiveness and lower risk of using ovarian tissue transplantation for cancer survivors.

\section{Selection of spermatozoa with fragmented DNA: practical recommendations}

Spermatozoa selection is one of the most important steps in IVF methods. Basic semen analyses and standard methods for sperm selection in many cases do not eliminate proportion of spermatozoa with genetic defects sufficiently. For spermatozoa selection there is currently a trend to combine multiple methods to separate inappropriate sperm cells based on multiple parameters to increase successful fertilization. How many methods are best combined, and whether a new Magnetic Activated Cell Sorting (MACS) selection method really brings a better prognosis in terms of conception success, has already been studied several times $[7,8]$. A study was conducted where the primary spermatozoa separation system used was the swim-up method, because it is a more gentle option that significantly decreased proportion of fragmented spermatozoa when compared to density gradient centrifugation [9]. This study compared the swim-up and MACS methods and their combinations. The swim-up and MACS treatments of spermatozoa were tested alone and in combinations where the first treatment was swim-up followed with MACS and vice versa. Using of the MACS method decreased proportion of spermatozoa with fragmented DNA. However, inclusion of the swim-up method before using the MACS is the most efficient and brings the greatest benefit for further use of sperm in the embryology laboratory in comparison with the other procedures. It is well known that the MACS has limitations regarding sperm concentration and volume for loading due to a small size and volume of its column. Therefore loading of fresh semen into the MACS column may reduce its filtering function and impede its ability to isolate motile non-apoptotic spermato- zoa, because dead/apoptotic sperm cells bind to the MACS column in competition with motile/non-apoptotic sperm cells. Based on the results of this study, utilization of the swim-up method followed by MACS separation can be recommended. This approach is efficient and can be advantageously used for separation of spermatozoa in assisted reproduction techniques. This combination of methods brings better results in sperm selection - lower proportion of spermatozoa with fragmented DNA and also, in comparison with MACS alone, better gains of total spermatozoa available for further IVF or ICSI methods.

\section{Artificial activation of oocytes}

The intracytoplasmic sperm injection (ICSI) is the most successful treatment method for male factor infertility quite independent on the sperm parameters. Despite its success rates, fertilization failure following ICSI still occurs in 1-3 \% of couples [10]. Such fertilization failure is due to unsuccessful oocyte activation, which could be sperm-borne or oocyte-borne [11].

Assisted oocyte activation (AOA) is being increasingly applied in human assisted reproduction to restore fertilization and pregnancy rates in couples with a history of ICSI fertilization failure [10]. The mostly used methods are the ionophore A23187 or ionomycin activation in a short time interval after ICSI.

Calcium ionophore treatment results in a statistically significant improvement of fertilization, blastulation, and implantation rates, as well as of pregnancy and live-birth rates. This demonstrates a strong positive effect of calcium ionophore use [12].

No significant difference was found between defect type (chromosomal aberrations) and malformation frequency or type, between the ICSI and AOA groups. No significant differences were found regarding birth weight, gestational week at time of delivery and fetal gender. Ionophore oocyte activation should be considered a legitimate option for cases of failed or low fertilization by ICSI [13].

In their study, the AOA using the ionophore A23187 in activation medium (Cult-Active Gynemed) was applied. Immediately after ICSI, the oocytes were immersed into $30 \mu \mathrm{l}$ drop of equilibrated activation medium for 15 minutes. Subsequently, the oocytes were repeatedly washed in culture medium and then cultured. Presence of two pronuclei and two polocytes was checked 18 hours later. The method was applied in 24 cycles of patients with previous ICSI failure. In 22 cycles, at least 2 oocytes were fertilized. The fertilization rate was $42 \%$ and 31 fresh/cryopreserved embryo transfers resulted in 11 pregnancies and 9 deliveries of a healthy newborn.

In our country, only a part of centers uses the AOA method. Last publications and our opinion support safety and effectiveness of the ionophore using method to be more widely used in the assisted reproduction. 


\section{Access of single and lesbian women to assisted reproduction technologies and possibility of their reproduction in the Czech Republic}

Although the Czech Republic has liberal laws allowing men and women to use the assisted reproduction technologies (ART) only on the basis of a written request regardless of their sexual orientation or current partner status, a heated discussion is going on as to whether this approach should be even more eased and a woman could be fertilized without a man's consent by sperm obtainable without any limitation from a sperm bank.

A conservative part of the society warns of an unregulated access to sperms in case of no donor's assurance which would make the state automatically accepting social-economical guarantees for the children. The supporters of unregulated access to gametes and its use in ART argue with: (a) the demographic aspects of declining birth rate in the sense that it depends on every child that may be born; (b) a human-legal opinion that every woman has the right to be a mother and therefore, when she wants a child, she has the right to have it; (c) a gender view according to which it is not admissible in developed society for a man to decide about a woman's body or her treatment and interfere in her body or approve her fertilization; (d) the regulation of unwanted, noting that current rules are anyway circumvented and forcing women to opt for an undignified way when searching for a partner who has to approve that he will accept a legitimate father's role. The advocates of current legislation object that: (a) the support of ART does not increase the fertility, women do not have more children not that they cannot have them but because they do not want to have more of them; (b) after reaching legal age, the citizens may enter into a marriage and start a family without any limitations, have children or undergo infertility treatment; (c) from a biological sense of reproduction it is natural that the bodies of a man and a woman are connected in the process of fertilization for which logically the involved man's consent is needed; (d) the laws must be met and argument that they are being bypassed anyway, does not stand. The access to ART should be determined by health indication, age, legislation, economical possibilities but also by social changes in population. Legislative norms intervening in human reproduction must be adopted on the basis of broad consensus and consideration of all biological-psycho-social aspects $[14,15]$.

\section{Conclusions}

A very fast progress was made in the field of assisted reproduction during the past decade. In 2014, approximately 1500 IVF cycles per million inhabitants were done in Europe, however in the Czech Republic it was more than 2500 cycles per million inhabitants. From this point of view, the Czech Republic is one of the leading countries in Europe (the $2^{\text {nd }}$ place after Denmark). It is due to a large number of citizens from Western Europe who arrive to the Czech Republic as IVF tourists. These trends support modern approaches using current knowledge. Czech IVF clinics offer medical and laboratory care at a high professional level.

This article brings several important topics which are spoken and solved in the Czech Republic. There is a number of other important issues like the "freeze-all" strategy, social freezing or surrogate motherhood, but it is not possible to include all these topics here. The presented topics are a selection of the most interesting from the $26^{\text {th }}$ Czech-Slovak symposium of assisted reproduction in 2017.

\section{Ethical approval:}

The conducted research is not related to either human or animals use.

\section{Acknowledgements}

Supported by the Ministry of Health, Czech Republic - conceptual development of research organization (FNBr, 65269705), project MSMT LTC 18059 and fund from the Faculty of Medicine MU to junior researcher Michal Jeseta. The authors are active participants of the COST Action CA16119 (In vitro 3-D total cell guidance and fitness)

\section{Corresponding author}

Ing. Ješeta Michal, Ph.D., Department of Obstetrics and Gynecology, University Hospital and Masaryk University, Obilni trh 11, 60200 Brno, Czech Republic, e-mail: Jeseta.Michal@fnbrno.cz

\section{Conflict of interest statement}

The authors declare they have no conflict of interest

\section{References}

1. Verberg MF, Macklon NS, Nargund G, Frydman R, Devroey P, Broekmans FJ, Fauser BC. Mild ovarian stimulation for IVF. Hum Reprod Update. 2009;15(1):13-29; DOI:10.1093/humupd/dmn056.

2. Fatemi H, Doody K, Griesinger G, Witjes H, Mannaerts B. High ovarian response does not jeopardize ongoing pregnancy rates and increases cumulative pregnancy rates in GnRH-antagonist protocol. Hum. Reprod. 2013;28(2):442-52; DOI:10.1093/humrep/des389.

3. Sunkara SK, Rittenberg V, Raine-Fenning N, Bhattacharya S, Zamora J, Coomarasamy A. Association between the number of eggs and live birth in IVF treatment: an analysis of 400135 treatment cycles. Hum Reprod. 2011;26(7):1768-74; DOI:10.1093/humrep/der106.

4. Youssef MA, van Wely M, Al-Inany H, Madani T, Jahangiri N, Khodabakhshi S, Alhalabi M, Akhondi M, Ansaripour S, Tokhmechy R, Zarandi L, Rizk A, El-Mohamedy M, Shaeer E, Khattab M, Mochtar MH, van der Veen F. A mild ovarian stimulation strategy in women with poor ovarian reserve undergoing IVF: a multicenter randomized non-inferiority trial. Hum Reprod. 2017;32(1):112-18; DOI:10.1093/humrep/dew282.

5. Pacheco F, Oktay K. Current Success and Efficiency of Autologous Ovarian Transplantation: A Meta-Analysis. Reprod Sci. 2017;24(8):111120; DOI:10.1177/1933719117702251.

6. Mardešić T, Jelínková L, Melicharová L. Successful ovarian tissue transplantation in woman with premature ovarian failure after gonadotoxic treatment. Ceska Gynekol. 2017;82(1):38-41.

7. Cakar Z, Cetinkaya B, Aras D, Ozkavukcu S, Kaplanoglu I, Cinar O. Does combining magnetic-activated cell sorting with density gradient or swim-up improve sperm selection? J Assist Reprod Genet. 2016;33(8):1059-65; DOI:10.1007/s10815-016-0742-0.

8. Chi HJ, Kwak SJ, Kim SG, Kim YY, Park JY, Yoo ChS, Park IH, Sun HG, Kim JW, Lee KH. Efficient isolation of sperm with high DNA integrity and stable chromatin packaging by a combination of density-gradient centrifugation and magnetic.activated cell sorting. Clin Exp Reprod Med. 2016;43(4):199-206; DOI:10.5653/cerm.2016.43.4.199.

9. Zini A, Finelli A, Phang D, Jarvi K. Influence of semen processing technique on human sperm DNA integrity. Urology. 2000;56(6):1081-4. 
10. Van den Meerschaut F, Nikiforaki D, Heindryckx B, De Sutter P. Assisted oocyte activation following ICSI fertilization failure. Reprod Biomed Online. 2014;28(5):560-71; DOI:10.1016/j.rbmo.2014.01.008.

11. Tesarik J, Rienzi L, Ubaldi F, Mendoza C, Greco E. Use of a modified intracytoplasmic sperm injection technique to overcome sperm-borne and oocyte-borne oocyte activation failures. Fertil Steril. 2002;78(3):61924; D0I:10.1016/S0015-0282(02)03291-0.

12. Murugesu S, Saso S, Jones BP, Bracewell-Milnes T, Athanasiou T, Mania A, Serhal P, Ben-Nagi J. Does the use of calcium ionophore during artificial oocyte activation demonstrate an effect on pregnancy rate? A meta-analysis. Fertil Steril. 2017,108(3):468-82; DOI:10.1016/j. fertnstert.2017.06.029.

13. Miller N, Biron-Shental T, Sukenik-Halevy R, Klement AH, Sharony R, Berkovitz A. Oocyte activation by calcium ionophore and congenital birth defects: a retrospective cohort study. Fertil Steril. 2016;106(3):590-6 DOI:10.1016/j.fertnstert.2016.04.025.

14. Konečná H. Parent at any time and anyway? $1^{\text {st }}$ ed. Praha: Mladá Fronta; 2017.

15. Shenfield F. Transgender and a parent: legal aspects. Precongress course 9: transgenderism and reproduction: State of the art in fertility options for transgender and people with sex reassignment. $33^{\text {rd }}$ Annual Meeting of ESHRE, Geneva, Switzerland, 2-5 July 2017. 